\title{
Gastrosquise: Diagnóstico Pré-natal x Prognóstico Neonatal
}

\author{
Gastroschisis: Prenatal Diagnosis x Neonatal Outcome \\ Melania Maria Ramos de Amorim, Paulo Carvalho Vilela, Luiz Carlos Santos \\ Gilliatt Hanois Falbo Neto, Luiz André Marinho Lippo \\ Marcelo Marques \\ RESUM0
}

\begin{abstract}
Objetivos: determinar a freqüência de diagnóstico pré-natal em recém-nascidos (RN) com gastrosquise operados no Instituto Materno-Infantil de Pernambuco (IMIP) e analisar suas repercussões sobre o prognóstico neonatal.

Métodos: realizou-se um corte transversal retrospectivo incluindo 31 casos de gastrosquise submetidos a correção cirúrgica em nosso Serviço entre 1995-1999. Calculou-se o risco de prevalência $(R P)$ de morte neonatal e seu intervalo de confiança a 95\% para a presença de diagnóstico pré-natal e outras variáveis cirúrgicas e perinatais, realizando-se análise de regressão logística múltipla para determinação do risco ajustado de morte neonatal.

Resultados: apenas 10 (32,3\%) dos 31 casos de gastrosquise tinham diagnóstico pré-natal e nasceram no IMIP. Nenhum RN com diagnóstico pré-natal foi prematuro, em contraste com $43 \%$ daqueles sem diagnóstico pré-natal $(p<0,05)$. O intervalo entre parto e correção cirúrgica foi maior na ausência (7,7 horas) que na presença de diagnóstico pré-natal (3,8 horas). $O$ tipo de cirurgia, a necessidade de ventilação mecânica assistida e a freqüência de infecção pós-operatória não apresentaram diferença significante entre os dois grupos. A mortalidade neonatal foi mais freqüente no grupo sem diagnóstico pré-natal (67\%) do que no grupo com diagnóstico pré-natal (20\%). Os outros fatores associados com risco aumentado de morte neonatal foram: idade gestacional <37 semanas, parto em outros hospitais, intervalo partocirurgia $>4$ horas, cirurgia em estágios, necessidade de ventilação mecânica e infecção.

Conclusões: o diagnóstico pré-natal foi infreqüente entre $R N$ com gastrosquise e a morte neonatal foi extremamente alta em sua ausência. É necessário aumentar a freqüencia de diagnóstico pré-natal e melhorar os cuidados perinatais para reduzir esta elevada mortalidade.
\end{abstract}

PALAVRAS-CHAVE: Gastrosquise. Diagnóstico pré-natal. Prognóstico neonatal. Malformações fetais. Prematuridade.

\section{Introdução}

A gastrosquise constitui um defeito da parede abdominal anterior, em geral situado à direita da inserção normal do cordão umbilical, medindo em torno de $2-5 \mathrm{~cm}$, e através do qual ocorre a herniação de diversas vísceras abdominais, porém mais freqüentemente de alças intestinais. Sua ocorrência varia de 1:10.000 a 1:15.000 partos, sendo mais rara que a da onfalocele. A incidência é maior em gestantes jovens e em

Instituto Materno-Infantil de Pernambuco (IMIP)

Correspondência:

Melania Maria Ramos de Amorim

Coordenação de Ensino do IMIP

Rua dos Coelhos, 300 - Ilha do Leite

50070-550 - Recife - PE conceptos do sexo masculino ${ }^{1-4}$.

Devido aos grandes avanços e à disseminação da ultra-sonografia na propedêutica da gestação nas duas últimas décadas, o diagnóstico de gastrosquise passou a ser possivel ainda no período pré-natal. Esta possibilidade desperta o interesse dos obstetras que, em conjunto com outros profissionais (especialistas em Medicina Fetal, cirurgiões pediátricos, neonatologistas), podem intervir na história natural desta anomalia, de forma a garantir um melhor prognóstico perinatal ${ }^{5-7}$.

O diagnóstico ecográfico é possivel a partir de 16-20 semanas, quando se constata o defeito da parede abdominal e alças intestinais flutuando livremente no líquido amniótico, sem membrana limitante. O volume herniado pode ser desproporcional ao pequeno tamanho da cavidade 
abdominal ${ }^{8,9}$. Com a ultra-sonografia endovaginal, o diagnóstico é possivel a partir de 12,5 semanas ${ }^{10}$. Anomalias estruturais e cromossômicas são raras, porém é freqüente a associação com malformações intestinais (atresias) ${ }^{11-13}$.

Durante o acompanhamento pré-natal, deve-se rastrear o retardo de crescimento intrauterino, que ocorre em 23-77\% dos $\operatorname{casos}^{9,11-16}$, bem como diagnosticar e inibir o trabalho de parto prematuro. A vitalidade fetal também deve ser monitorizada, pelo risco de sofrimento fetal ${ }^{17,18}$. Existem controvérsias quando à via de parto e à idade gestacional ideal para interrupção da gestação. Embora alguns autores defendam a cesárea eletiva pré-termo ${ }^{19}$ para profilaxia da exposição prolongada do conteúdo abdominal ao líquido amniótico e prevenção do comprometimento das alças intestinais, diversos outros estudos têm demonstrado resultados perinatais semelhantes para o parto vaginal e a cesárea ${ }^{13}$, 20-22. Além disso, parece que o parto prematuro terapêutico não está associado a diminuição na necessidade de utilização do silo (prótese de silicone que recobre as alças, permitindo sua redução progressiva para a cavidade abdominal e o fechamento em estágios), início mais precoce de alimentação oral ou encurtamento da permanência hospitalar ${ }^{21,23}$. Na revisão de Simmons e Georgeson $^{23}$, inclusive, o maior percentual de colocação do silo e uma hospitalização prolongada foram mais freqüentes nos recém-nascidos de parto prematuro entre 35-37 semanas do que nos recém-nascidos de termo ${ }^{23}$.

A maior parte dos autores sugere, atualmente, que o parto nas gestações complicadas por gastrosquise deve ocorrer a termo, a fim de minimizar as conseqüências deletérias da prematuridade, que podem agravar o prognóstico e, inclusive, retardar ou impedir a correção cirúrgica $^{21,23}$. Assim, salvo na presença de grave retardo do crescimento, de sofrimento fetal ou outra intercorrência obstétrica relevante, não há indicações para o parto prematuro terapêutico ${ }^{7,9,21,23}$.

$\mathrm{Na}$ verdade, o fundamental na assistência ao parto na presença de gastrosquise é que este deve ocorrer em centros de referência, com o apoio da equipe multidisciplinar (Obstetrícia, Neonatologia, Cirurgia Pediátrica) e com estrutura de unidade de terapia intensiva para o atendimento dos recém-nascidos afetados ${ }^{9,24,25}$. O transporte intra-útero oferece resultados infinitamente melhores que a transferência do neonato, que pode levar a comprometimento das condições clínicas (hipotermia, desidratação) e do aspecto das alças ${ }^{24,25}$. A correção cirúrgica deve ser realizada o mais rapidamente possível, tendo já sido demonstrado que o prognóstico é tanto melhor quanto menor o intervalo entre o parto e a cirurgia ${ }^{25,26}$.

Evidentemente, esta abordagem só é possível quando se consegue efetuar o correto diagnóstico pré-natal, encaminhando-se a gestante aos hospitais com assistência terciária. Em nosso hospital, lidamos todavia com uma realidade bem diversa, pois apesar de a freqüência de diagnóstico pré-natal ter aumentado nos últimos anos, a maior parte dos casos de gastrosquise operados no serviço de Cirurgia Pediátrica do IMIP não foram identificados durante a gestação. Assim, estamos lidando em geral com recém-nascidos cujo parto foi assistido em outros hospitais, tanto do Recife como de outras cidades, sendo freqüente o transporte inadequado e sua admissão em condições clínicas desfavoráveis.

O presente estudo foi realizado com o objetivo de determinar a freqüência do diagnóstico prénatal em recém-nascidos com gastrosquise operados no IMIP e o impacto desse diagnóstico sobre o prognóstico, analisando os principais fatores associados à ocorrência de óbito neonatal.

\section{Pacientes e Métodos}

Realizou-se um estudo descritivo, retrospectivo, tipo coorte transversal, englobando 31 casos de gastrosquise operados no IMIP entre janeiro de 1995 e dezembro de 1999. Diagnosticou-se gastrosquise quando o recém-nascido apresentava defeito da parede abdominal à direita da inserção normal do cordão umbilical, de diâmetro variável, com herniação do conteúdo abdominal, em geral alças intestinais ${ }^{27}$.

$\mathrm{Na}$ análise inicial, considerou-se como variável independente (preditora) a presença ou não de diagnóstico pré-natal, e como variáveis dependentes (resposta) os seguintes fatores: local do parto (no IMIP ou em outro hospital de Recife ou outras cidades), tipo de parto (transvaginal ou cesárea), idade gestacional e peso ao nascer, intervalo entre o parto e a correção cirúrgica, estado geral na admissão ao serviço de Cirurgia Pediátrica, tipo de correção cirúrgica, necessidade ou não de ventilação mecânica assistida (VMA) e nutrição parenteral total (NPT), infecção, óbito neonatal e tempo de permanência hospitalar.

Em segunda etapa da análise, a ocorrência ou não de óbito neonatal foi considerada como variável dependente e todas as outras variáveis passaram a ser tratadas como independentes.

O diagnóstico pré-natal da gastrosquise é realizado a partir da detecção, por ultra- 
sonografia antenatal, de alças livres flutuando no líquido amniótico, sem membrana limitante ${ }^{27}$. A conduta anteparto compreende a monitorização do bem-estar fetal (perfil biofísico fetal e cardiotocografia), prevenção da prematuridade (tocólise, se necessário) e corticoterapia para aceleração da maturidade pulmonar fetal. Todos os casos com diagnóstico pré-natal são discutidos pela equipe multidisciplinar (obstetras, neonatologistas, cirurgiões pediátricos e intensivistas). A interrupção é indicada apenas depois de se atingir o termo da gestação (37 semanas), indicando-se em geral a via alta (operação cesariana), com a presença do cirurgião pediátrico na sala de parto ${ }^{27}$.

A conduta pós-natal adotada pelo Serviço de Cirurgia Pediátrica do IMIP nos casos de gastrosquise baseia-se fundamentalmente na correção cirúrgica, que é realizada o mais rápido possível após o nascimento, desde que as condições clínicas do recém-nascido sejam favoráveis. Recém-nascidos provenientes de outros hospitais e cidades freqüentemente chegam ao serviço com estado geral comprometido, hipotermia e desidratação, e nesses casos tratam-se inicialmente estas intercorrências e realiza-se a cirurgia após estabilização do quadro clínico. A abordagem cirúrgica compreende o fechamento primário ou em estágios (com colocação do silo e fechamento secundário), de acordo com a decisão do cirurgião, baseado na quantidade e no aspecto do intestino eviscerado. Os cuidados pós-operatórios incluem antibioticoterapia (com penicilina e gentamicina), observação em unidade de terapia intensiva (UTI), ventilação mecânica assistida e nutrição parenteral, quando necessário, programando-se a alta em caso de evolução favorável com função respiratória normal e alimentação oral (leite materno) bem-sucedida.

Para as finalidades do presente estudo, a idade gestacional foi determinada pelo método somático-neurológico de Capurro et al. ${ }^{28}$, devido às dificuldades para se obter a data da última menstruação em diversas pacientes que não tiveram parto assistido no IMIP nem tinham ultrassonografia precoce. Consideraram-se prematuros os recém-nascidos com menos de 37 semanas calculadas por este método. O peso ao nascer foi expresso em gramas e codificado como baixo peso quando inferior a 2.500 gramas. Os escores de Apgar não puderam ser analisados devido à falta de informação nos casos transferidos de outros serviços.

O intervalo entre o parto e a correção cirúrgica foi determinado para todos os recém-nascidos e expresso em horas. O estado geral do recémnascido logo após o nascimento (partos assistidos no IMIP) ou na admissão no serviço de Cirurgia Pediátrica foi considerado como bom, regular ou mau, de acordo com a avaliação subjetiva pelo cirurgião assistente. Avaliou-se a correção cirúrgica, se realizada em tempo único (fechamento primário) ou em estágios (colocação do silo e reparo secundário). Determinou-se o número de dias em ventilação mecânica assistida e nutrição parenteral total, quando estas foram indicadas. Infecção neonatal foi definida a partir de critérios clínicos (comprometimento do estado geral, ictericia, anemia, sinais respiratórios, taquicardia, hepatoesplenomegalia, alterações do tônus e reflexos, convulsão, vômitos, diarréia e distensão abdominal) e laboratoriais. A duração do internamento foi calculada como o intervalo entre a correção cirúrgica e o egresso (alta ou óbito).

A análise estatística foi realizada utilizando-se o programa de dominio público Epi-Info 6.04b e o SPSS 6.0 para Windows. Utilizou-se o teste de Mann-Whitney para comparação das variáveis dependentes quantitativas e os testes $\chi^{2}$ de associação (Pearson) e exato de Fisher para as variáveis categóricas, de acordo com a presença ou não de diagnóstico pré-natal. Considerou-se estatisticamente significativo um erro alfa $(\mathrm{p})$ menor que $5 \%$. Calculou-se o risco de prevalência (RP) de morte neonatal para as diversas variáveis independentes, bem como seu intervalo de confiança a 95\%. Regressão logística múltipla foi realizada para controlar o efeito das variáveis potencialmente confundidoras sobre a associação diagnóstico pré-natal x óbito neonatal. Nesta etapa, as variáveis foram codificadas binariamente como $1=\operatorname{sim}$ ou 0 = não, e utilizou-se o modelo Forward: Conditional ou Stepwise de regressão logística, no programa SPSS para Windows.

\section{Resultados}

Dos 31 casos de gastrosquise operados no periodo de janeiro de 1995 a dezembro de 1999, apenas $10(32,3 \%)$ tinham diagnóstico pré-natal. Verificou-se uma nítida associação entre diagnóstico pré-natal e local do parto, uma vez que todos os casos com diagnóstico pré-natal tiveram o parto assistido no IMIP e quase todos os recém-nascidos sem diagnóstico pré-natal eram provenientes de outros serviços.

A freqüência de cesárea foi significativamente maior na presença de diagnóstico pré-natal (90\% versus $24 \%$ ), uma vez que a maior parte dos casos com diagnóstico pré-natal teve o parto programado no IMIP, excetuando-se uma paciente na qual desencadeou-se o trabalho de par- 
to com rápida evolução para parto transpélvico. Não foram encontrados casos de recém-nascidos prematuros no grupo com diagnóstico prénatal, ao passo que a freqüência de prematuridade foi de $43 \%$ na ausência deste diagnóstico (média de idade gestacional de 38,2 e 36 semanas, respectivamente). Embora o peso ao nascer tenha sido mais elevado nos recém-nascidos com diagnóstico pré-natal (2.688 gramas versus 2.454 gramas) e a freqüência de baixo peso (menor que 2.500 gramas) tenha sido maior quando não havia diagnóstico pré-natal $(52 \%$ versus $30 \%$ ), essa diferença não foi estatisticamente significativa $(\mathrm{p}>0,05)$ (Tabela 1$)$.

\begin{tabular}{|c|c|c|c|}
\hline \multirow{2}{*}{$\begin{array}{l}\text { Resultados Obstétricos e } \\
\text { Perinatais }\end{array}$} & \multicolumn{2}{|c|}{ Diagnóstico Pré-Natal } & \multirow[t]{2}{*}{$p$} \\
\hline & $\begin{array}{l}\text { Presente } \\
n=10\end{array}$ & $\begin{array}{c}\text { Ausente } \\
n=21\end{array}$ & \\
\hline \multicolumn{4}{|l|}{ Tipo de parto } \\
\hline Vaginal & $1(10 \%)$ & $16(76 \%)$ & 0,0007 \\
\hline Cesárea & $9(90 \%)$ & $5(24 \%)$ & \\
\hline \multicolumn{4}{|l|}{ Idade gestacional (Capurro) } \\
\hline$<37$ semanas & - & $9(43 \%)$ & 0,01 \\
\hline$\geq 37$ semanas & $10(100 \%)$ & $12(57 \%)$ & \\
\hline Média \pm DP & $38,2 \pm 1,1$ & $36,0 / 2,9$ & 0,04 \\
\hline \multicolumn{4}{|l|}{ Peso ao nascer } \\
\hline$<2.500$ gramas & $03(30 \%)$ & $11(52 \%)$ & 0,2 \\
\hline$\geq 2.500$ gramas & $7(70 \%)$ & $10(48 \%)$ & \\
\hline Média \pm DP & $2.688 \pm 543$ & $2.454 / 463$ & 0,1 \\
\hline
\end{tabular}

Analisando-se as condições clinicas pré-cirúrgicas, observou-se que recém-nascidos com diagnóstico pré-natal tinham boas condições clínicas, contra apenas 33\% dos recém-nascidos sem diagnóstico pré-natal, diferença esta que foi estatisticamente significativa. Em relação ao intervalo parto-correção cirúrgica, a média foi de 3,8 horas na presença de diagnóstico pré-natal e de 7,2 horas em sua ausência. Essa correção foi realizada até 4 horas depois do parto em $70 \%$ e $24 \%$ dos casos, de acordo com a presença ou não de diagnóstico pré-natal, respectivamente ( $p$ $<0,05)$. Não houve diferença estatisticamente significativa quanto ao tipo de cirurgia, se em tempo único (reparo primário) ou em estágios (silo) em relação ao diagnóstico pré-natal, apesar de a correção em tempo único ter sido mais freqüente na presença de diagnóstico pré-natal $160 \%$ versus 43\%) (Tabela 2).
Tabela 2 - Comparação de variáveis pré-operatórias e tipo de cirurgia de acordo com a presença ou não de diagnóstico pré-natal em casos de gastrosquise operados no Serviço de Cirurgia Pediátrica do IMIP.

\begin{tabular}{lccc}
\hline Variáveis & \multicolumn{2}{c}{ Diagnóstico Pré-Natal } & p \\
& $\begin{array}{c}\text { Presente } \\
\mathbf{n = 1 0}\end{array}$ & $\begin{array}{c}\text { Ausente } \\
\mathbf{n}=\mathbf{2 1}\end{array}$ & \\
\hline $\begin{array}{l}\text { Condições clínicas } \\
\text { Boas }\end{array}$ & $9(90 \%)$ & $7(33 \%)$ & 0,004 \\
Regulares/ruins & $1(10 \%)$ & $14(77 \%)$ & \\
Intervalo parto-cirurgia & & & \\
$\leq 4$ horas & $7(70 \%)$ & $5(24 \%)$ & 0,02 \\
$>4$ horas & $3(30 \%)$ & $16(76 \%)$ & \\
Média \pm DP & $3,8 \pm 3,2$ & $7,7 \pm 3,7$ & 0,006 \\
Tipo de Cirurgia & & & \\
Tempo único & $6(60 \%)$ & $12(43 \%)$ & 0,6 \\
Em estágios & $4(40 \%)$ & $9(57 \%)$ & \\
\hline
\end{tabular}

A necessidade de ventilação mecânica assistida foi de $67 \%$ para os recém-nascidos sem diagnóstico pré-natal e $40 \%$ para os recém-nascidos com diagnóstico pré-natal, porém essa diferença não foi estatisticamente significativa. A duração da nutrição parenteral foi significativamente mais elevada na ausência em relação à presença de diagnóstico pré-natal (respectivamente 14,2 e 7,2 dias). A duração do internamento também foi maior na ausência de diagnóstico pré-natal (18,8 versus 12,7 dias), mas sem significância estatística. A freqüência de infecção pós-operatória foi de $50 \%$ na presença e $71 \%$ na ausência de diagnóstico pré-natal ( $p>0,05)$. Analisando-se a freqüência de óbito, esta foi significativamente maior no grupo sem diagnóstico pré-natal, em torno de $67 \%$, comparada a $20 \%$ no grupo com diagnóstico pré-natal (Tabela 3). A principal causa de óbito foi a infecção (responsável por 93\% das mortes neonatais).

Analisando-se a associação entre as diversas variáveis independentes e o óbito neonatal, observou-se um risco de prevalência significativamente maior para idade gestacional menor que 37 semanas (todos os prematuros morreram), peso ao nascer menor que 2.500 gramas, parto em outro hospital (fora do IMIP), intervalo entre o parto e a correção cirúrgica maior que 4 horas, estado geral regular ou comprometido na avaliação préoperatória, correção cirúrgica em estágios, necessidade de ventilação mecânica assistida e infecção pós-operatória. Esse risco foi significativamente diminuido quando havia diagnóstico pré-natal, porém permaneceu inalterado para o tipo de parto (vaginal ou cesárea) e necessidade de nutrição parenteral (Tabela 4). 
Tabela 3 - Evolução pós-operatória de acordo com a presença ou não de diagnóstico pré-natal em casos de gastrosquise operados no Serviço de Cirurgia Pediátrica do IMIP.

\begin{tabular}{lccc}
\hline Variáveis & \multicolumn{2}{c}{ Diagnóstico Pré-Natal } & $\mathbf{p}$ \\
& $\begin{array}{c}\text { Presente } \\
\mathbf{n}=10\end{array}$ & $\begin{array}{c}\text { Ausente } \\
\mathbf{n}=\mathbf{2 1}\end{array}$ & \\
& & & \\
Necessidade de ventilação & & & \\
mecânica assistida & $4(40 \%)$ & $14(67 \%)$ & 0,15 \\
Sim & $6(60 \%)$ & $7(33 \%)$ & \\
Não & $7,2 \pm 4,9$ & $14,2 \pm 10,2$ & 0,04 \\
Duração de nutrição parenteral & & & \\
(Média \pm DP) - em dias & & & \\
Infecção pós-operatória & & $15(71 \%)$ & 0,2 \\
Sim & $5(50 \%)$ & $6(29 \%)$ & \\
Não & $5(50 \%)$ & & \\
Duração do internamento & $12,7 \pm 8,9$ & $18,8 \pm 10,7$ & 0,16 \\
(Média \pm DP) & & & \\
Resultado Final & & & \\
Alta & $8(80 \%)$ & $7(33 \%)$ & 0,02 \\
Óbito & $2(20 \%)$ & $14(67 \%)$ & \\
\hline
\end{tabular}

Quando se realizou análise de regressão logística múltipla para controle das variáveis potencialmente confundidoras, verificou-se que as variáveis que demonstraram mais forte associação com o óbito neonatal foram o peso ao nascer menor que 2.500 gramas, o intervalo entre parto e cirurgia maior que 4 horas e a necessidade de ventilação mecânica assistida $(\mathrm{p}<0,05)$.

\section{Discussão}

Apenas 35\% dos recém-nascidos com gastrosquise operados em nosso serviço tinham diagnóstico pré-natal estabelecido pela ultrasonografia. Essa baixa freqüência de diagnóstico pré-natal pode ser atribuída ao fato de que a maior parte dos casos (65\%) eram provenientes de outros hospitais, do Recife ou de outras cidades de Pernambuco. A transferência após o parto para o IMIP tem ocorrido porque este é um hospital terciário dispondo de leitos de Obstetricia, Cirurgia Pediátrica e unidade de terapia intensiva pediátrica, e constitui portanto centro de referência para as diversas malformações congênitas. Esse resultado diferencia-se bastante da freqüência descrita na literatura internacional, uma vez que quase todas as casuísticas estudadas referemse a países desenvolvidos nos quais a ultrasonografia faz parte da rotina pré-natal e a transferência dos casos de gastrosquise é realizada com o concepto intra-útero ${ }^{25,26,29,30}$.
Tabela 4 -Risco de prevalência de morte neonatal de acordo com variáveis perinatais e cirúrgicas.

\begin{tabular}{|c|c|c|c|c|}
\hline \multirow[t]{2}{*}{ Variáveis } & \multicolumn{2}{|c|}{ Morte neonatal } & \multirow{2}{*}{$\begin{array}{c}\text { Risco de } \\
\text { prevalência }\end{array}$} & \multirow[t]{2}{*}{ IC- $95 \%$} \\
\hline & $\mathrm{n}$ & $\%$ & & \\
\hline \multicolumn{5}{|l|}{ Diagnóstico pré-natal } \\
\hline Ausente $(n=21)$ & 14 & 67 & 1,00 & \\
\hline Presente $(n=10)$ & 2 & 20 & 0,30 & $0,08-1,00$ \\
\hline \multicolumn{5}{|l|}{ Idade gestacional } \\
\hline$\geq 37$ semanas $(n=22)$ & 7 & 32 & 1,00 & \\
\hline$<37$ semanas $(n=9)$ & 9 & 100 & 3,14 & $1,70-5,79$ \\
\hline \multicolumn{5}{|l|}{ Tipo de parto } \\
\hline Cesariana $(n=14)$ & 6 & 43 & 1,00 & \\
\hline Transvaginal $(n=17)$ & 10 & 59 & 1,37 & $0,67-2,83$ \\
\hline \multicolumn{5}{|l|}{ Local do parto } \\
\hline $\operatorname{IMIP}(n=11)$ & 3 & 27 & 1,00 & \\
\hline Outros hospitais $(n=20)$ & 13 & 65 & 2,38 & $0,86-6,59$ \\
\hline \multicolumn{5}{|c|}{ Peso ao nascer } \\
\hline$\geq 2500$ gramas $(n=17)$ & 5 & 29 & 1,00 & \\
\hline$<2500$ gramas $(n=14)$ & 11 & 79 & 2,67 & $1,22-5,86$ \\
\hline \multicolumn{5}{|l|}{ Estado geral } \\
\hline $\operatorname{Bom}(n=16)$ & 3 & 19 & 1,00 & \\
\hline Regular/mau $(n=15)$ & 13 & 87 & 4,62 & $1,64-13,07$ \\
\hline \multicolumn{5}{|l|}{ Intervalo parto-cirurgia } \\
\hline$<4$ horas $(n=11)$ & 2 & & 1,00 & \\
\hline$\geq 4$ horas $(n=20)$ & 14 & 70 & 3,85 & $1,06-13,93$ \\
\hline \multicolumn{5}{|l|}{ Tipo de cirurgia } \\
\hline Tempo único $(n=13)$ & 4 & 31 & 1,00 & \\
\hline Em estágios ( $n=18$ ) & 12 & 67 & 2,17 & $0,90-5,22$ \\
\hline \multicolumn{5}{|l|}{ Ventilação mecânica assistida } \\
\hline Não (n = 13) & 2 & 15 & 1,00 & \\
\hline $\operatorname{Sim}(n=18)$ & 14 & 78 & 5,06 & $1,38-18,52$ \\
\hline \multicolumn{5}{|l|}{ Nutrição parenteral } \\
\hline Não (n = 5) & 3 & 60 & 1,00 & \\
\hline $\operatorname{Sim}(n=16)$ & 13 & 81 & 0,83 & $0,37-1,88$ \\
\hline \multicolumn{5}{|l|}{ Infecção } \\
\hline Não (n = 11) & 1 & 9 & 1,00 & \\
\hline $\operatorname{Sim}(n=20)$ & 15 & 75 & 8,25 & $1,25-54,38$ \\
\hline
\end{tabular}

Comparando-se as diversas características dos recém-nascidos com gastrosquise de acordo com a presença ou não de diagnóstico prénatal, encontraram-se importantes diferenças que resultaram em uma mortalidade significativamente mais elevada na ausência de diagnóstico pré-natal.

A maior parte dos casos sem diagnóstico prénatal $(95 \%)$ teve o parto assistido em outro serviço, ao passo que o parto daqueles com diagnóstico pré-natal sempre ocorreu no IMIP, sendo em ge- 
ral programado (90\% de cesarianas). Assim, o intervalo entre o parto e a correção cirúrgica foi significativamente mais elevado no grupo sem diagnóstico pré-natal (média de 7,7 horas versus 3,8 horas quando havia diagnóstico pré-natal). Por outro lado, enquanto todos os recém-nascidos com diagnóstico pré-natal tinham idade gestacional a partir de 37 semanas, 43\% dos recém-nascidos sem diagnóstico pré-natal eram prematuros (média de idade gestacional em torno de 36 semanas). Não foi surpresa, portanto, encontrar-se uma mortalidade 3 vezes maior nos casos sem diagnóstico pré-natal (67\% versus 20\%), e uma mortalidade global superior a $50 \%$, que destoam nitidamente das taxas de óbito descritas na última década na maioria dos países desenvolvidos, inferior a $10 \%{ }^{29,30}$ e só se aproximam da mortalidade por gastrosquise na Nigéria, superior a $60 \%{ }^{31}$. No Brasil, um estudo realizado na Universidade de Campinas (São Paulo) por Sbragia Neto et al. ${ }^{32}$ encontrou uma mortalidade global de $32 \%$, com maior freqüência de óbitos no grupo sem diagnóstico prénatal $(54,5 \%)$ em relação aos recém-nascidos com diagnóstico pré-natal (14,3\%). Mesmo com uma mortalidade menor do que a verificada em nossa casuística, esse estudo também concluiu que o diagnóstico pré-natal contribuía favoravelmente para o atendimento perinatal adequado às crianças com gastrosquise. Essa conclusão reveste-se de peculiar importância em se tratando de países em desenvolvimento, como o nosso, onde o parto dos recém-nascidos sem diagnóstico pré-natal em geral ocorre em hospitais sem estrutura de cuidados neonatais intensivos, requerendo-se o transporte destes sem o minimo de condições e sem adequada proteção das alças intestinais. Às vezes percorrem-se longas distâncias, com agravamento das condições clínicas dos recém-nascidos, maior freqüência de hipotermia e desidratação e conseqüente piora do prognóstico neonatal. Embora o tipo de parto mais adequado persista ainda controverso em casos de gastrosquise, diversos autores pontificam que os melhores resultados ocorrem quando, independente da via (cesárea ou vaginal), o parto ocorre em centros de referência, dotados de infra-estrutura de cuidados intensivos e sob atenção imediata da equipe multidisciplinar ${ }^{9,24,25,33}$. Não creditamos portanto o melhor prognóstico nos casos com diagnóstico pré-natal à via de parto, mesmo porque foram realizadas diversas cesáreas por outras indicações no grupo sem diagnóstico pré-natal e não houve associação entre tipo de parto e óbito neonatal.

No entanto, o parto programado dentro do nosso serviço facilitou a imediata assistência ao recém-nascido, coordenando-se a equipe médica e permitindo iniciar-se a correção cirúrgica o mais rápido possivel. Na verdade, nos últimos casos aqui assistidos, a cirurgia da gastrosquise foi realizada logo após o nascimento, em sala cirúrgica vizinha à da cesárea, e os casos em que se postergou a correção cirúrgica deveram-se unicamente à necessidade de estabilização do neonato quando estavam presentes alterações respiratórias como a taquipnéia transitória.

Esse menor intervalo entre parto e correção cirúrgica reveste-se de fundamental importância, pois evita a formação da matriz gelatinosa (peel) que reveste as alças intestinais expostas na gastrosquise e previne-se a deterioração do estado geral do recém-nascido ${ }^{9,26,34}$. Além disso, o intervalo aumentado em nossa casuística nos casos sem diagnóstico pré-natal deveu-se sobretudo ao tempo prolongado transcorrido entre o parto em outro serviço, muitas vezes em outras cidades, e a transferência do recém-nascido em condições inadequadas, sem a preocupação com o aquecimento e a proteção das alças. Em conseqüência, a grande maioria destes chegou ao nosso hospital em condições clínicas desfavoráveis, com desidratação, hipotermia e deterioração do aspecto do intestino exteriorizado.

É importante ressaltar, outrossim, a elevada freqüência de prematuridade no grupo sem diagnóstico pré-natal, contrastando com a exclusividade de recém-nascidos de termo na presença de diagnóstico pré-natal. Convém lembrar que a prematuridade é bastante freqüente em casos de gastrosquise, apresentando importante associação com o mau prognóstico neonatal ${ }^{9,16,23}$. No presente estudo, inclusive, todos os recém-nascidos prematuros morreram. Esse fato pode ser atribuido tanto às complicações específicas da prematuridade, como a síndrome de desconforto respiratório do recém-nascido (SDRN), como à maior susceptibilidade do prematuro com gastrosquise à hipotermia, desidratação e complicações locais (intestinais), decorrentes do transporte inadequado (nenhum deles foi transferido em incubadora) e do maior intervalo entre o nascimento e a admissão na unidade neonatal de cuidados intensivos.

Entretanto, sabendo-se que o trabalho de parto prematuro ocorre em cerca de 30\% das gestações complicadas por gastrosquise, um importante objetivo da assistência pré-natal, quando se estabelece o diagnóstico intra-útero desta malformação, é evitar a prematuridade, o que pode ser obtido por meio da identificação precoce do incremento da contratilidade uterina e do emprego de drogas tocoliticas ${ }^{9,27,35}$. Além disso, a terapia corticóide é empregada rotineiramente quando se sabe que o feto tem gastrosquise, e mesmo que acabe por ocorrer o parto prematuro, a incidência de SDRN reduz-se em aproxi- 
madamente $50 \%$, enquanto são prevenidas também outras complicações, como a hemorragia intraventricular, enterocolite necrotizante e persistência do ductus arteriosus ${ }^{36}$.

Um achado interessante é que, apesar de a idade gestacional ter sido significativamente mais elevada entre os recém-nascidos com diagnóstico pré-natal, o peso ao nascer não apresentou diferença estatisticamente significativa em relação aos recém-nascidos sem diagnóstico prénatal. É claro que existe a possibilidade de que o pequeno tamanho da amostra tenha impedido evidenciar-se uma diferença que na realidade exista. No entanto, é válido supor que, como o retardo de crescimento ocorre freqüentemente em fetos com gastrosquise ${ }^{9}$, talvez o peso ao nascer não aumente significativamente com o maior tempo de permanência intra-útero, apesar dos diversos outros beneficios acarretados pelo prolongamento da gravidez.

Apesar de diversas outras variáveis poderem estar associadas aos resultados adversos observados neste estudo quando não se realizou o diagnóstico pré-natal, talvez devido ao pequeno tamanho da amostra, esta associação não pôde ser demonstrada (erro tipo II). A necessidade de ventilação mecânica assistida e de nutrição parenteral total têm sido referidas como fatores de risco para óbito, no entanto na presente série não se demonstrou associação significativa destas variáveis com o diagnóstico pré-natal, da mesma forma que a correção cirúrgica em estágios. Conforme já analisamos, na ausência de diagnóstico pré-natal o parto ocorre em locais distantes e sem infraestrutura de atendimento aos recém-nascidos com gastrosquise, que são então transferidos, demorando várias horas até que se possa realizar a correção cirúrgica. Como não apenas o estado geral mas as próprias alças intestinais têm suas condições agravadas, pode não ser factivel o reparo primário, requerendo-se o fechamento em estágios, com colocação do silo ${ }^{37}$. Adicionalmente, é mais comum requerer-se a ventilação mecânica assistida no pós-operatório e, pelo íleo adinâmico persistindo por vários dias por conta das alterações intestinais, instituir-se nutrição parenteral prolongada. Todas essas intercorrências, além da fragilidade característica dos prematuros, sobrelevam o risco de infecção, que em nossa casuística representou a principal causa de morte nos casos de gastrosquise.

Quando realizamos análise de regressão logística múltipla para determinar as variáveis mais fortemente associadas ao óbito, observouse um risco ajustado significativamente maior para o peso ao nascer menor que 2.500 gramas, o intervalo entre parto e correção cirúrgica mai- or que 4 horas e a realização de ventilação mecânica assistida. Prematuridade e infecção acabaram por ser retiradas do modelo pelo fato de que representavam, mais do que fatores de risco, a própria causa do óbito neonatal. Uma vez que o peso ao nascer se associa à prematuridade (que pode ser prevenida), que o intervalo parto-correção cirúrgica pode ser diminuído se todos os nascimentos passarem a ser assistidos em hospitais de referência e a própria ventilação mecânica pode ter sua indicação reduzida com os cuidados adequados e imediatos ao recémnascido com gastrosquise, conclui-se que parte significativa desses óbitos poderia ter sido evitada caso se tivesse previamente o diagnóstico fetal, com cuidados pré-natais, intraparto e neonatais adequados.

Embora tenhamos intenção de prosseguir prospectivamente este estudo, englobando maior número de casos e controlando diversas outras variáveis que podem exercer um efeito confundidor, acreditamos que os dramáticos resultados encontrados devem ser de imediato divulgados, visando à adoção de medidas preventivas. Apontamos todavia que a grande maioria destas medidas independe da conduta adotada em nosso Serviço, requerendo antes a intervenção do próprio Sistema de Saúde, porque dizem respeito fundamentalmente à qualidade da assistência pré-natal e ao correto funcionamento dos serviços de referência e contra-referência. Somente quando se conseguir um diagnóstico pré-natal correto não apenas de gastrosquise como de outras malformações é que poderemos prestar o adequado atendimento às gestações com estas complicações e providenciar o transporte intra-uterino, para que o parto ocorra em hospitais terciários, com todas as facilidades para a correção cirúrgica e os cuidados intensivos aos recém-nascidos.

\section{SUMMARY}

Purpose: to determine the frequency of prenatal diagnosis in newborns with gastroschisis operated at the Instituto Materno-Infantil de Pernambuco (IMIP) and to analyze its repercussions on neonatal prognosis.

Patients and Methods: a cross-sectional study was carried out, including 31 cases of gastroschisis submitted to surgical correction in our Service from 1995 to 1999. Prevalence risk $(P R)$ of neonatal death and its $95 \%$ confidence interval were calculated for the presence of prenatal diagnosis and other perinatal and surgical variables. Multiple logistic regression analysis was carried out to determine the adjusted risk of neonatal death.

Results: only 10 of 31 cases of gastroschisis (32.3\%) had 
prenatal diagnosis and all were delivered at IMIP. No newborn with prenatal diagnosis was preterm but $43 \%$ of those without prenatal diagnosis were premature $(p<0,05)$. Birth-to-surgery interval was significantly greater in the absence of prenatal diagnosis ( 7.7 versus 3.8 hours). The type of surgery, need of mechanical ventilation and frequency of postoperative infection were not different between the groups. Neonatal death was more frequent in the group without prenatal diagnosis (67\%) than in the group with prenatal diagnosis (20\%). The main factors associated with increased risk of neonatal death were gestational age $<37$ weeks, absence of prenatal diagnosis, delivery in other hospitals, birth-to-surgery interval $>4$ hours, staged silo surgery, need of mechanical ventilation and postoperative infection.

Conclusions: prenatal diagnosis was infrequent among infants with gastroschisis and neonatal death was extremely high in its absence. It is necessary to achieve greater rates of prenatal diagnosis and to improve perinatal care in order to reduce this increased mortality.

KEY WORDS: Gastroschisis. Prenatal diagnosis. Neonatal outcome. Fetal malformations. Prematurity.

\section{Referências}

1. Moore TC, Nur K. An international survey of gastroschisis and omphalocele (490 cases). II. Relative incidence, pregnancy and environmental factors. Pediatr Surg Int 1986; 1:105-9.

2. Roeper PJ, Harris J, Lee G, Neutra R. Secular rates and correlates for gastroschisis in California (1968 - 1977). Teratology 1987; 35:203-10.

3. Goldbaum G, Daling J, Milham S. Risk factors for gastroschisis. Teratology 1990; 42:397-403.

4. Calzolari E, Volpato S, Bianchi F, et al. Omphalocele and gastroschisis: a collaborative study of five Italian congenital malformation registries. Teratology 1993; 47:47-55.

5. D'Alton ME, DeCherney AH. Prenatal diagnosis. N Engl J Med 1993; 328:114-20.

6. Crombleholme TM, D'Alton M, Cendron M, et al. Prenatal diagnosis and the pediatric surgeon: the impact of prenatal consultation on perinatal management. J Pediatr Surg 1996; 31:156-63.

7. Isfer EV, Sanchez R, Silva MM. Aparelho digestivo e parede abdominal. In: Isfer EV, Sanchez R, Saito, M, editores. Medicina Fetal. $1^{\mathrm{a}}$ ed. Rio de Janeiro: Revinter; 1996. p. 118-63.

8. Martin RW. Screening for fetal abdominal wall defects. Obstet Gynecol Clin North Am 1998; 25:517-26.

9. Paidas MJ, Crombleholme TM, Robertson RM. Prenatal diagnosis and management of the fetus with an abdominal wall defect. Semin Perinatol 1994; 18:196-214.

10.Guzman ER. Early prenatal diagnosis of gastroschisis with transvaginal ultrasonography. Am J Obstet Gynecol 1990; 162:1253-4.

11. Moore TC, Nur K. An international survey of gastroschisis and omphalocele (490 cases). I. Nature and distribution of additional malformations. Pediatr Surg Int 1986; 1:46-50.

12.Nicolaides KH, Snijders RJM, Cheng HH, Gosden C. Fetal gastro-intestinal and abdominal wall defects: Associated malformations and chromosomal abnormalities. Fetal Diagn Ther 1992; 7:102-15.

13.Novotny DA, Klein RJ, Boeckman CR. Gastroschisis: an 18-year review. J Pediatr Surg 1993; 28:650-2.

14. Carpenter MW, Curci MR, Dibbins AW, Haddow JE. Perinatal management of ventral wall defects. Obstet Gynecol 1984; 64:646-51.

15. Raynor BD, Richards D. Growth retardation in fetuses with gastroschisis. J Ultrasound Med $1997 ; 16: 13-6$.

16. Molenaar JC, Tibboel D. Gastroschisis and omphalocele. World J Surg 1993; 17:337-41.

17.Burge DM, Ade-Ajayi N. Adverse outcome after prenatal diagnosis of gastroschisis: the role of fetal monitoring. J Pediatr Surg 1997; 32:441-4.

18.Crawford RA, Ryan G, Wright VM, Rodeck CH. The importance of serial biophysical assessment of fetal wellbeing in gastroschisis. $\mathrm{Br} \mathrm{J}$ Obstet Gynaecol 1992; 99:899-902.

19. Moore TC, Collins DL, Catanzarite V, Hatch EL Jr. Pre-term and particularly pre-labor cesarean section to avoid complications of gastroschisis. Pediatr Surg Int 1999; 15:97-104.

20.Moretti M, Khoury A, Rodriquez J, Lobe T, Shaver $D$, Sibai B. The effect of mode of delivery on the perinatal outcome in fetuses with abdominal wall defects. Am J Obstet Gynecol 1990; 163:833-8.

21.Adra AM, Landy HJ, Nahmias J, Gómez-Marin O. The fetus with gastroschisis: Impact of route of delivery and prenatal ultrasonography. Am J Obstet Gynecol 1996; 174:540-6.

22.Quirk JG Jr, Fortney J, Collins HB 2nd, West J, Hassad SJ, Wagner C. Outcomes of newborns with gastroschisis: The effects of mode of delivery, site of delivery, and interval from birth to surgery. Am J Obstet Gynecol 1996; 174:1134-40.

23.Simmons M, Georgeson KE. The effect of gestational age at birth on morbidity in patients with gastroschisis. J Pediatr Surg 1996; 31:1060-2.

24.Nicholls G, Upadhyaya V, Gornall P, Buick RG, Corkery JJ. Is specialist center delivery of gastroschisis beneficial? Arch Dis Child 1993; 69:71-3. 
25. Stoodley N, Sharma A, Noblett H, James D. Influence of place of delivery on outcome of babies with gastroschisis. Arch Dis Child 1993; 68:321-3.

26.Coughlin JP, Drucker DE, Jewell MR, Evans MJ, Klein MD. Delivery room repair of gastroschisis. Surgery 1993; 114:822-7.

27.Amorim MMR, Vilela PC, Santos LC, Falbo GH. Onfalocele e gastrosquise: diagnóstico e conduta pré-natal. Rev IMIP 1999; 13:56-65.

28. Capurro H, Konichezky S, Fonseca D, CaldeyroBarcia R. A simplified method for diagnosis of gestational age in the newborn infant. $\mathrm{J}$ Pediatr 1978; 93:120-2.

29. Rinehart BK, Terrone DA, Isler CM, Larmon JE, Perry KG Jr, Roberts WE. Modern obstetric management and outcome of infants with gastroschisis. Obstet Gynecol 1999; 94:112-6.

30.Ortiz VN, Villarreal DH, González Olmo J, Ramos Perea C. Gastroschisis: a ten year review. Bol Asoc Med P R 1998; 90:69-73.

31.Agugua NE, Nwako FA. Gastroschisis a fifteen-year experience. West Afr J Med 1990; 9:147-50.
32. Sbragia Neto L, Melo Filho AA, Barini R, Hughet PR, Marba S, Bustorff-Silva JM. Importância do diagnóstico pré-natal de gastrosquise. Rev Bras Ginecol Obstet 1999; 21:475-9.

33.Langer JC. Gastroschisis and Omphalocele. Semin Pediatr Surg 1996; 5:124-8.

34.Langer JC, Longaker MT, Crombleholme TM, et al. Etiology of intestinal damage in gastroschisis. I. Effects of amniotic fluid exposure and bowel constriction in a fetal lamb model. J Pediatr Surg 1989; 24:992-7.

35. Mercer BM, Lewis R Preterm labor and preterm premature rupture of the membranes. Diagnosis and management. Infect Dis Clin North Am 1997; $11: 177-201$

36. Crowley PA. Corticosteroids prior to preterm delivery. In: Neilson JP, Crowther CA, Hodnett ED, Hofmeyr GJ, editors. Pregnancy and childbirth module of the Cochrane database of systematic reviews. Oxford: Update Software; 1997. (The Cochrane Collaboration, Issue 4)

37.Stringer MD, Brereton RJ, Wright VM. Controversies in the management of gastroschisis: a study of 40 patients. Arch Dis Child 1991; 66:34-6.

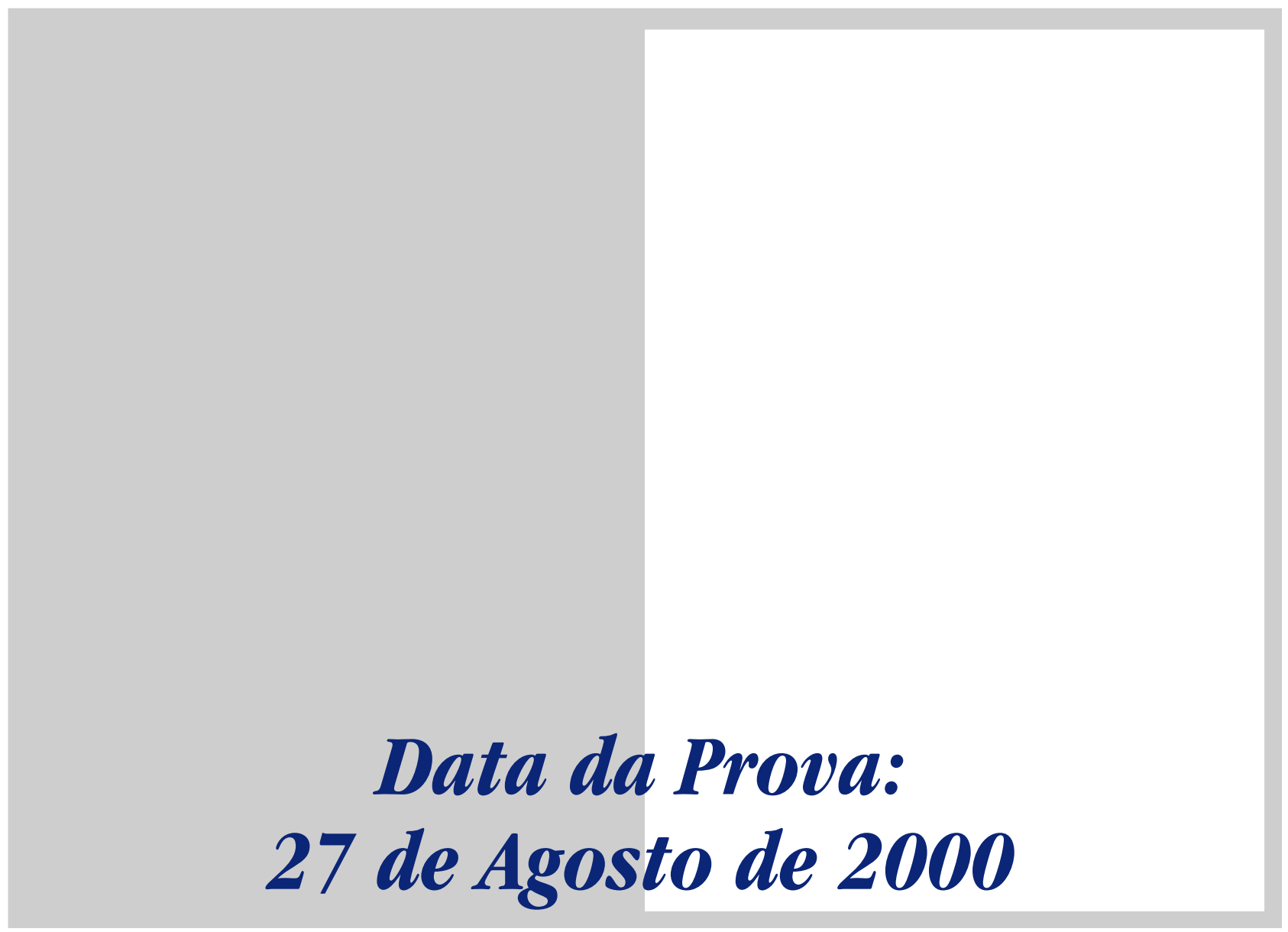

